El presente artículo hace hincapié en que las deficiencias factuales son relativamente frecuentes en toda clase de textos didácticos y divulgativos y que, en el marco de la traducción comunicativa, es necesario que el traductor proceda a su detección en el texto de partida y a su corrección en el texto de llegada. En relación con la presencia de un importante error factual en un libro divulgativo de biología evolutiva de Oxford University Press, se distinguen tres categorías de traductores de textos científico-técnicos didácticos y divulgativos en función de la facilidad y de los recursos con que pueden detectar (y subsanar) tales defectos textuales: el traductor especialista, el semiespecialista y el no especialista.

PALABRAS CLAVE: enseñanza y divulgación de la ciencia; error factual en el texto de partida; traducción científico-técnica; traducción comunicativa.

\title{
El traductor no especialista ante la presencia de deficiencias factuales en el texto de partida didáctico o divulgativo: un estudio de caso sobre lo ideal, lo suficiente y lo inaceptable
}

The Non-Specialist Translator as confronted with the Occurrence of Factual Errors in an Instructional or Popularizing Source Text: a Case Study on the Ideal, the Sufficient, and the Unacceptable

The following article emphasizes that factual errors are relatively frequent in all sorts of instructional and popularizing texts, and that within the framework of communicative translation, it is necessary for the translator to spot those defects in the source text and to rectify them in the target text. Focusing on an important factual error present in a popularizing book on evolutionary biology published by Oxford University Press, three categories of translators of instruc-

CARlos GARrido tional and popularizing texts with scientific-technical content are distinguished according to the ease and to the resources with which Universidade de Vigo they can detect (and rectify) such textual flaws, i. e., the specialist translator, the semi-specialist translator, and the non-specialist translator.

KEY WORDS: communicative translation; science education and popularization; source-text factual error; scientific and technical translation. 


\section{INTRODUCCIÓN}

Los textos científico-técnicos incluyen, con cierta frecuencia, deficiencias factuales y, cuando son objeto de traducción contemporánea, sus traductores, siguiendo las pautas propias de la traducción instrumental, o comunicativa, deben esforzarse en no trasladar al texto de llegada, corrigiéndolas en este, tales contravenciones o erosiones de la función informativa imperante en la comunicación pragmática y especializada (Schmitt, I999a: 59-6r; Schmitt, I999b: I48; Horn-Helf, I999: I63; Fleischmann y Schmitt, 2004: 536; Garrido, 2015a; Garrido, 2015b; Garrido, 2016: 357-358, 4II-4I3). A este respecto, consideremos el siguiente caso, que ilustra claramente los dos aspectos que se acaban de referir, el error factual presente en el texto de partida científico-técnico y su corrección en el texto de llegada. En la oración final de un artículo de tema médico publicado en agosto de 2015 en la revista de (alta) divulgación científica Scientific American, su autor, el profesor de otorrinolaringología $\mathrm{M}$. Charles Liberman, afirmaba:

[ra] Scientific American, 8/2015: 43 (subrayado nuestro): «An otologist may one day be able to deliver drugs to the cochlea using a minimally invasive treatment for noise-induced ear damage as easily as an ophthalmologist corrects a myopic eye by laser surgery of the lens.»

En este pasaje, pues, se expresa la esperanza de que en un futuro sea posible tratar la sordera inducida por el ruido recurriendo a una administración de medicamentos a la cóclea tan sencilla como la corrección de la miopía que hoy se efectúa mediante una operación con láser del cristalino (en el original inglés, «by laser surgery of the lens»). En realidad, aquí se ha insinuado un error, pues tal corrección quirúrgica de la

miopía, como incluso bastantes legos en medicina saben, no se efectúa en el cristalino (EN lens), sino en la córnea (EN cornea), confusión de palabras que el atento traductor de este artículo para Investigación y Ciencia (versión española de Scientific American) no reproduce en su texto de llegada:

[rb] Investigación y Ciencia, го/2015: 69 (subrayado nuestro): «Tal vez algún día un otólogo podrá aplicar fármacos en la cóclea mediante un tratamiento mínimamente invasivo contra el daño auditivo causado por el ruido con la misma facilidad con la que un oftalmólogo corrige la miopía con cirugía láser de la córnea».

Como vemos en este ejemplo, el traductor debe contar con la posible presencia de deficiencias factuales en su texto de partida científico-técnico incluso en el caso de que este sea un texto publicado, sometido a procesos de revisión editorial y editado por una publicación de tanto prestigio como la estadounidense Scientific American. En este sentido, basándonos en nuestra experiencia como lectores y traductores de textos destinados a la enseñanza y divulgación de la ciencia, creemos que la apreciación de Peter A. Schmitt que transcribimos a continuación, aunque pueda resultar verdadera en términos relativos - cuando se comparan todos los géneros textuales científico-técnicos-, en lo concerniente a la presencia de defectos en los libros didácticos y artículos y libros de divulgación del ámbito científico-técnico, se revela demasiado optimista:

Bei Normen und Patentschriften - die im Zuge des Normierungs- bzw. Patentverfahrens mehrmals von verschiedenen Personen besonders sorgfaltig geprüft werden, sowohl hinsichtlich formaler Korrektheit als 
auch hinsichtlich fachlicher (technischer/ juristischer) Richtigkeit [...] - liegen trozt umfangreicher Textcorpora keine Belege über Defekte vor. Sehr gering ist auch die Fehlerwahrscheinlichkeit in Lehrbüchern. Populärwissenschaftliche Zeitschriften wie z.B. Scientific American, National Geographic, Geo scheinen - so mein über Jahre hinweg gewonnener Eindruck - ebenfalls sehr sorgfältig redigiert zu sein [...]. (Schmitt, I999b: $\left.\mathrm{I} 47^{-\mathrm{I}} 48\right)^{\mathrm{I}}$

En este contexto, y a falta de una investigación empírica que sería interesante realizar en el futuro, el presente artículo efectúa, a continuación (apartado 2), un estudio de caso que analiza las posibilidades de detección, y de corrección en el texto de llegada, de deficiencias factuales presentes en textos científicos didácticos y divulgativos por parte de un traductor competente, aunque no especialista en la correspondiente (sub)disciplina, así como los recursos conceptuales y documentales de que aquel puede y debe valerse para superar el desafío en cuestión. Además, en relación con la figura del traductor especialista, en el apartado 3, el presente trabajo pone de manifiesto una actitud nada ejemplar frecuentemente mostrada por traductores de esa condición cuando vierten textos científicos didácticos o divulgativos.

\footnotetext{
I Nuestra traducción: «En el caso de las normas técnicas y de las patentes de invención, las cuales en el curso de los respectivos procesos de elaboración y registro son revisadas de manera especialmente cuidadosa múltiples veces por parte de diversas personas, tanto desde el punto de vista de la corrección formal como desde el punto de vista de su rigor conceptual (técnico y jurídico) [...], no consta noticia alguna de deficiencias, a pesar de los extensos corpora textuales que de ellas están disponibles. También muy pequeña es la probabilidad de error en el caso de los libros de texto, e igualmente rigurosa parece - esa es mi impresión, obtenida a lo largo de años- la redacción de las revistas de divulgación científica, como Scientific American, National Geographic o Geo [...].».
}

\section{ESTUDIO DE CASO: POSIBILIDADES} Y RECURSOS DEL TRADUCTOR NO ESPECIALISTA FRENTE A LAS DEFICIENCIAS FACTUALES DEL TEXTO DE PARTIDA DESTINADO A LA ENSEÑANZA O DIVULGACIÓN DE LA CIENCIA

El presente estudio de caso se refiere a una deficiencia factual, de importancia no desdeñable en su contexto, que surge en un libro de divulgación científica del campo de la biología elaborado por dos reputados especialistas en genética. A seguir, en el apartado 2.I, se presentan en pormenor esta obra, sus autores y el defecto textual en cuestión, y en los subapartados del apartado 2.2 se analizan las características, las correspondientes posibilidades de actuación frente al error factual del texto base y los recursos conceptuales y documentales propios de tres categorías de traductores corrientemente involucrados en traducciones de esta naturaleza, categorías definidas en función de la mayor o menor especialización disciplinar del traductor.

\section{Presentación del texto (de partida), de sus autores y de la deficiencia factual}

El texto que contiene el error factual que aquí vamos a analizar se titula Evolution: A Very Short Introduction y fue publicado en 2003 por la prestigiosa Oxford University Press en su serie de libros divulgativos «Very Short Introductions», de la que constituye el número Ioo. Esta serie de divulgación, integrada por libros de pequeño formato y de alrededor de I5o páginas, escritos por renombrados especialistas, ofrece una síntesis simultáneamente accesible y rigurosa de una gran diversidad de temas y disciplinas y se ha revelado un enorme éxito editorial y cultural, como atestigua el gran número de volúmenes ya editados y de lenguas a que algunos títulos de la colección han sido vertidos: 
VERY SHORT INTRODUCTIONS are for anyone wanting a stimulating and accessible way into a new subject. They are written by experts and have been translated into more than 40 different languages. The series began in 1995 and now covers a wide variety of topics in every discipline. The vsi library now contains over 400 volumes-a Very Short Introduction to everything from Psychology and Philosophy of Science to American History and Relativity - and continues to grow in every subject area. (Nota editorial presente en el volumen n. ${ }^{\circ} 450$ [año 2015] de la colección «Very Short Introductions»)

El volumen Evolution: A Very Short Introduction - destinado, como todos los de la colección, a un público adulto no especialista (en biología), aunque culto y con curiosidad científica- ofrece una síntesis de los conocimientos actuales sobre el fenómeno de la evolución biológica, de modo que la obra, a lo largo de ocho capítulos y 145 páginas (con 2I ilustraciones en blanco y negro y unas pocas tablas y esquemas), presenta los fundamentos del proceso evolutivo, pasa revista a la diversidad de pruebas que evidencian la existencia de evolución (por un lado, semejanzas y diferencias constatables entre organismos y, por otro, distribución de los seres vivos en el espacio y en el tiempo), explica los mecanismos de la adaptación y de la selección natural, así como la formación y divergencia de especies, y concluye con la exposición de algunas cuestiones evolutivas de especial complejidad. ${ }^{2}$

2 Aunque, como se ha afirmado, los volúmenes de la colección «Very Short Introductions» de la Oxford University Press han sido objeto de traducción a un gran número de lenguas, en concreto la obra que aquí nos interesa, Evolution: A Very Short Introduction, no ha sido traducida, todavía, en España, como indica la base de datos española del ISBN (‘www.mcu.es/webISBN〉), ni en Portugal, según la Bibliografia Nacional Portuguesa (http: //bibliografia. bnportugal.pt/bnp/bnp.exes), consultadas en internet a I0.5.2017.
Los autores de Evolution: A Very Short Introduction son los esposos Brian Charlesworth y Deborah Charlesworth, dos reputados biólogos británicos que gozan de amplio reconocimiento internacional. Brian Charlesworth (nacido en I945) es especialista en genética de poblaciones, ha investigado, sobre todo, la evolución y selección natural de secuencias moleculares, regenta la cátedra de Biología Evolutiva de la Universidad de Edimburgo (Escocia) y, desde I99I, es fellow de la Royal Society of London, cuya Darwin Medal ganó en 2000. A su vez, Deborah Charlesworth (nacida en 1943) es destacada especialista en genética evolutiva de las plantas, investigadora de la Universidad de Edimburgo y, desde 2005, fellow de la Royal Society of London.

Si las apreciaciones hechas en los párrafos anteriores, correspondientes a unas condiciones editoriales de gran calidad y a unos autores de reconocido prestigio, pueden ayudar a calibrar, en vista del defecto textual que en breve expondremos, la importancia de que el traductor se prevenga contra la eventualidad de la presencia de deficiencias factuales en su texto de partida didáctico o divulgativo, en el mismo sentido se deberán tomar las siguientes consideraciones, relativas a la intervención de revisores textuales. En efecto, el original de Evolution: A Very Short Introduction, según se puede leer en su capítulo de agradecimientos (pág. viII) 3 y en el breve texto laudatorio de su portada posterior, ${ }^{4}$ fue revisado, por lo menos, por cuatro personas,

3 «We also thank Helen Borthwick, Jane Charlesworth, and John Maynard Smith for reading and commenting on the first draft of the manuscript. All remaining errors are, of course, our fault».

4 Apreciación de Richard Dawkins sobre el libro impresa en la portada posterior: «But if you want a summary of what is known to be true about evolution today, and how we know it, you will find none more agreeably succinct than this vigorous little book». 
entre las cuales se encuentran dos grandes figuras de la biología evolutiva contemporánea: el genético John Maynard Smith (1920-2004) y el etólogo Richard Dawkins (nacido en I94I).

En cuanto al defecto textual, de naturaleza factual (no formal), presente en Evolution: $A$ Very Short Introduction que vamos a analizar en este trabajo, téngase en cuenta que surge en la página 5 del libro, en el capítulo 3, titulado «The evidence for evolution: similarities and differences between organisms»:

[2a] Evolution: A Very Short Introduction: I5 $_{5}$ (subrayados nuestros): "Another set of facts that strongly supports the theory of evolution is provided by modifications of the same structure in different species. For instance, the bones of bats' and birds' wings indicate clearly that they are modified forelimbs, even though they look very different from the forelimbs of other vertebrates (Figure IB). Similarly, although the flippers of whales look much like fish fin, and are clearly also well adapted for swimming, their internal structure is like the feet of other mammals, except for an increased number of digits. This makes sense, given all the other evidence that whales are modified mammals (for instance, they breathe with lungs and suckle their young). Fossil evidence shows that the two pairs of limbs of land vertebrates are derived from the two pairs of fins of the lobe-finned fishes (of which coelacanths are the most famous living representatives, see Chapter 4). Indeed, the earliest land vertebrate fossils had more than five digits on their limbs, just like fishes and whales.»

A continuación, of recemos nuestra traducción «literal» al castellano, con reproducción de la designación original, de ese fragmento textual:

[2b] Nuestra traducción «literal» (subrayados nuestros): «Otra serie de hechos que respal- dan fuertemente la teoría de la evolución la proporcionan las modificaciones que sufre una misma estructura en especies distintas. Así, por ejemplo, los huesos de las alas de los murciélagos y de las aves indican claramente que se trata de extremidades anteriores modificadas, por mucho que su aspecto sea muy diferente del de las extremidades anteriores de otros vertebrados (ilustr. IB). Del mismo modo, aunque las aletas pectorales de los cetáceos se parecen mucho a las de los peces, y también están, claramente, bien adaptadas para nadar, su estructura interna es como la de las patas de otros mamíferos, excepción hecha de su mayor número de dedos, lo cual tiene sentido, considerando todas las otras circunstancias que evidencian que los cetáceos son mamíferos modificados (por ejemplo, respiran mediante pulmones y amamantan a las crías). Las pruebas fósiles muestran que los dos pares de extremidades de los vertebrados terrestres derivan de los dos pares de aletas de los sarcopterigios (cuyos representantes vivos más célebres son los celacantos: v. cap. 4). De hecho, los fósiles de los vertebrados terrestres más antiguos testimonian que estos tenían más de cinco dedos en las extremidades, justamente como sucede en los peces y en los cetáceos.)

Teniendo en cuenta que, como veremos en los testimonios aportados en las próximas secciones, los tetrápodos (anfibios, reptiles, aves y mamíferos) actuales, cuando no se tienen en cuenta casos patológicos o aberrantes (polidactilia de individuos aislados), presentan siempre en sus extremidades un máximo de cinco dedos (pentadactilia como condición primitiva en los tetrápodos actuales), y que en las extremidades (anteriores) de los cetáceos se verifica una adaptación consistente en el incremento evolutivo del número de falanges (biperfalangia) en algunos de sus cinco dedos (o, en unas pocas especies, por reducción secundaria, de sus cuatro dedos), se puede concluir que el pasaje de Evolution: $A$ 
Very Short Introduction que se acaba de transcribir incluye un defecto textual que, de acuerdo con la clasificación de Garrido (2015a: 459-46r, 120 462-463; 20I6: 357-380, 389-406, 4II-4I3), cabe calificar, en principio (mas v. infra sección 3), de deficiencia factual de tipo "lapso léxico», pues, aquí, tal error parece derivar de la circunstancia de que los redactores han utilizado, por descuido, la palabra digits 'dedos', en vez de la correcta phalanges 'falanges'. 5

\section{Posibilidades y recursos del traductor no especialista en el tratamiento de deficiencias factuales del texto de partida didáctico o divulgativo}

Con el objetivo de reflexionar sobre el comportamiento que, frente a las deficiencias factuales del texto de partida, puede y debe mostrar un traductor no especialista en la disciplina a que se adscribe el correspondiente texto base didáctico o divulgativo, en este apartado vamos a tomar en consideración, y en relación con la deficiencia factual presente en el pasaje textual [2a], ya explicada, las posibilidades de desempeño y los recursos conceptuales y documentales, en primer lugar, de un traductor especialista (subapartado 2.2.I), en segundo lugar, de un traductor semiespecialista (2.2.2), y, por último (2.2.3), de un traductor no especialista.

5 Puesto en comunicación el autor de este trabajo con el Prof. Brian Charlesworth a través del correo electrónico, para advertirlo de este error y para facilitar su corrección con vistas a una eventual segunda edición de la obra, el autor principal de Evolution: A Very Short Introduction respondió (a 24.4.20I7) reconociendo el lapso y agradeciendo la advertencia: «Dear Prof. Garrido, You are of course correct. I am not sure how this error crept in. We are supposed to be producing a second edition (although the publishers are moving very slowly), and we will make sure it is removed. Best regards, Brian Charlesworth».

\section{Comportamiento del traductor especialista}

Reconocemos aquí como traductor especialista de un determinado texto científico-técnico a aquel traductor que dispone de formación específica, y de los correspondientes conocimientos especializados, en la disciplina, o incluso subdisciplina, a que se adscribe el texto de partida en cuestión. Cuando este traductor también dispone de una sólida formación (reglada) y experiencia en el campo de la traducción, nos encontramos en la situación ideal para afrontar actos de traducción como el que aquí nos ocupa. Por consiguiente, en nuestro caso concreto, un traductor tal de Evolution: A Very Short Introduction dispondría, aproximadamente, de la formación y de los conocimientos propios de un biólogo evolutivo. En concreto, estas condiciones ideales, óptimas, las cumplen, en general, los traductores de Investigación y Ciencia, encargados de verter al castellano los artículos divulgativos de Scientific American, pues se trata de especialistas en las respectivas disciplinas científicas (profesores, investigadores) que presentan buena competencia traductora (v. supra ej. [ra,b]).

Sin embargo, en relación con lo expuesto en el párrafo anterior han de introducirse dos matizaciones importantes. En primer lugar, si bien el perfil del traductor antes trazado es el que se revela ideal u óptimo para abordar la traducción de textos científico-técnicos, por un lado, el gran número de traducciones científicotécnicas, sobre todo de textos divulgativos, que entre nosotros es necesario efectuar torna insuficiente el (reducido) contingente de tales traductores, y, por otro lado, ese perfil de traductor ideal no es imprescindible, en la práctica, como aquí veremos, para garantizar la calidad de las traducciones de textos científico-técnicos de carácter didáctico o divulgativo. En segundo lugar, ha de tenerse en cuenta que los textos científico-técnicos didácticos y divulgativos, en 
contraste con los de investigación, por su enfoque integrador o sintetizador, suelen abarcar, no una única (sub)disciplina, sino varias (sub) disciplinas de un determinado campo — cuando no varios campos de especialidad-, por lo que, en la práctica, el traductor ideal antes descrito (especialista estricto solo en una determinada [sub]disciplina), a lo largo de la traducción de un texto didáctico o divulgativo podrá verse confrontado por desafíos similares a los del traductor semiespecialista o a los del traductor no especialista, que caracterizaremos en los siguientes apartados. Así, en nuestro caso concreto, si la disciplina a la que se puede adscribir Evolution: $A$ Very Short Introduction es la biología evolutiva (en realidad, una superdisciplina), también cabe decir que, a lo largo de la obra, van aflorando asuntos y datos propios de diversas (sub)disciplinas (bioquímica, genética, fisiología, botánica [sistemática], zoología [sistemática], ecología, etc.), y, de hecho, el pasaje [2a], que contiene el error factual aquí analizado, corresponde a la (sub)disciplina zoología (de vertebrados).

Configurada de este modo la condición del traductor especialista, puede admitirse que este, ante una afirmación del texto de partida del tipo de «los cetáceos poseen más de cinco dedos en sus extremidades (anteriores)» (ej. [2a]), con elevada probabilidad advertirá su falsedad, su carácter contrafactual, de forma inmediata (al hilo de una primera lectura), o, por lo menos, con elevada probabilidad será embargado por la fuerte sospecha —que deberá confirmar en una consulta documental- de que tal afirmación es errónea. En este caso, la probabilidad de que se dé una detección inmediata del error factual, o de que la sospecha de falsedad sea profunda, será mayor cuando el traductor especializado lo sea, no solo en la correspondiente disciplina (en nuestro caso, la biología evolutiva), sino también en la subdisciplina en cada momento abordada (en el pasaje [2a], la zoología [de vertebrados]).

A continuación, aducimos una serie de enunciados extraídos de la bibliografía zoológica (aquí, escrita en varias lenguas extranjeras [fuentes, estas, que, en algunos casos, suplen las lagunas de la bibliografía española]) para mostrar el acervo de elementos conceptuales (y de unidades léxicas de especialidad) que, durante la lectura de la afirmación falsa de [2a] relativa a los cetáceos (que son tetrápodos modernos), un traductor especialista, con elevada probabilidad, puede movilizar de forma inmediata o, por lo menos, a los que puede recurrir de manera provechosa mediante la consulta de la fuente correspondiente (fuentes que pueden ser altamente especializadas: obsérvense, a continuación, los términos grupo troncal y grupo de copa, que corresponden a conocimientos avanzados de sistemática [zoológica]):

Die Extremitäten der Tetrapoden sind ursprünglich fünfstrahlig (pentadactyl). (Kyrieleis y Paulus, 2000) ${ }^{6}$

Die in der Stammgruppe der Tetrapoda zunächst variable Anzahl der Finger- und Zehenstrahlen wurde auf fünf reduziert (Acanthostega† besaß 8, Ichthyostega† 7, Tulerpeton $\dagger$ 6). (Mickoleit, 2004: 232)7

Die Zahl der Finger und Zehen ist bei den Stammgruppen-Tetrapoden noch variabel und wird erst mit der Kronengruppe auf

6 Nuestra traducción: «Las extremidades de los tetrápodos muestran primitivamente cinco radios digitales (son pentadáctilas)».

7 Nuestra traducción: «El número de radios digitales de pies y manos, en un principio variable en el seno del grupo troncal de los Tetrápodos, se redujo finalmente a cinco ( $†$ Acanthostega poseía $8 ; \dagger I$ chthyostega, $7, \mathrm{y} \dagger$ Tulerpeton, 6)». 
maximal fünf Strahlen festgelegt (Pentadactylie) [...]. (Schultze y Schoch, 2004: 305 $)^{8}$

Cetaceans (whales, dolphins, and porpoises) have a soft tissue flipper that encases most of the forelimb, and elongated digits with an increased number of phalanges (hyperphalangy). In addition, some cetaceans exhibit a reduction in digit number. Although toothed cetaceans (odontocetes) are pentadactylous, most baleen whales (mysticetes) are tetradactylous and also lack a metacarpal. [...] Most mysticete (baleen whale) lineages (Balaenopteridae, Neobalaenidae, and Eschrichtiidae) also [como algunos ictiosaurios] only have four digital rays, each consisting of a metacarpal and some phalanges [...]. (Cooper et al., 2007: 654, 656)

De hecho, un traductor especialista en zoología de vertebrados (subdisciplina) podría, incluso, conocer, sin consulta previa, la circunstancia de que en el pasado geológico existió un grupo de tetrápodos que, en algunos casos, como condición derivada, sí presentaba (¡en contraste con los cetáceos!) extremidades con un número de dedos superior a cinco (biperdactilia), los ictiosaurios:

Les Ichthyosaures (planche 6.27, figures E-G) ont conservé les membres postérieurs mais montrent une transformation encore plus poussée en palette natatoire que les Cétacés. Chez les formes du Crétacé (planche 6.27, figure $\mathrm{G}$ ), seul le stylopode court et trapu conserve une individualité. Tous les autres éléments du membre, de forme polygonale, constituent une sorte de mosaïque avec byperphalangie manifeste et souvent hyperdactylie. (Beaumont y Cassier, 1987: 223-224)

8 Nuestra traducción: «En los tetrápodos del grupo troncal, el número de dedos de manos y pies es aún variable y no quedará fijado, en un máximo de cinco radios digitales, hasta el grupo de copa (pentadactilia) [...]».
The resemblance of the pectoral fin to an arm is very striking in an early tichthyosaur (†Mixosaurus, of the Middle Triassic). All of the individual elements, including the pisiform, can be recognized. Only five fingers are present, but the number of phalanges has increased in the anterior fingers, a condition called polyphalangy. The individual elements are shorter and more closely united in a late $\dagger$ ichthyosaur ( $\uparrow$ Ichthyosaurus from the Lower Jurassic). The numbers [sic] of digits has increased (polydactyly), as has the number of phalanges. (Liem et al, 200I: 306)

Der spindelförmige Körper [de los ictiosaurios] ähnelte dem der Delphine und die Extremitäten waren zu Flossen umgebildet. Sie hatten jegliche Beweglichket in den Gelenken verloren und zeichneten sich durch eine Vermehrung der Phalangen (Hyperphalangie) und teilweise der Finger (Polydactylie) aus. (Sander, 2004: 352)9

\section{Comportamiento del traductor semiespecialista} Podemos considerar como circunstancia, si no ideal u óptima, sí muy favorable el hecho de que un traductor, aunque no sea especialista en la (sub)disciplina a que se adscribe el texto de partida didáctico o divulgador, sí sea buen conocedor del correspondiente campo o rama científica, condición que denominamos de semiespecialista (pudiendo el traductor, entonces, ser especialista en otra disciplina de ese campo). Así, en nuestro caso, un traductor bastante aventajado de Evolution: A Very Short Introduction sería aquel que, no siendo especialista en biología evolutiva (ni en ninguna subdisciplina de esa disciplina), sí dispusiese de buenos cono-

9 Nuestra traducción: «El cuerpo [de los ictiosaurios], fusiforme, se asemejaba al de los delfines y las extremidades estaban transformadas en aletas. Estas habían perdido toda movilidad en las articulaciones y se caracterizaban por un aumento del número de falanges (biperfalangia) y, en algunos casos, del número de dedos (biperdactilia)». 
cimientos de biología en general, como sucede con un licenciado o graduado en Biología.

Trazado de ese modo el perfil del traductor semiespecialista, puede admitirse que, ante una afirmación del texto de partida del tipo de «los cetáceos poseen más de cinco dedos en sus extremidades (anteriores)» (ej. [2a]), es algo probable que aquel advierta de inmediato (al hilo de una primera lectura) su carácter falaz, o, por lo menos, es muy probable que le asalte la sospecha - que deberá confirmar en una consulta documental- de que tal afirmación es errónea.

Sobre todo en este último supuesto (sospecha), el traductor semiespecialista podrá manejar elementos conceptuales y recurrir a fuentes (de especialización intermedia) como las aducidas a continuación, que ya se refieren, todas ellas, directamente a los cetáceos:

Encore de type pinnipède chez les Siréniens, le membre devient une palette falciforme chez les Cétacés par hyperphalangie des doigts II et III (respectivement I3 et 7 phalanges chez le Globicéphale) et réduction des doigts latéraux I, IV e V (planche 6.27, figures $\mathrm{C}$ et $\mathrm{D}$ [en esta ilustración, que muestra el esqueleto del miembro anterior izquierdo de un delfín, se ven 5 dígitos en la mano o autopodio]). (Beaumont y Cassier, 1987: 222-224)

[Caracteres constitutivos de los Cetáceos:] • Die Vorderextremitäten sind zu Steuerflossen (Flipper) umgebildet (Abb. 623 [en esta ilustración, que muestra la cintura escapular y el esqueleto braquial de un delfín, se ven 5 dígitos en la mano o autopodio]). Die Fingerstrahlen sind äußerlich nicht mehr erkennbar. [...] - Hyperphalangie (Phalangenvermehrung). Der zweite Finger ist in der Regel der längste (Abb. 623b). (Mickoleit, 2004: 558)

Io Nuestra traducción: «[Caracteres constitutivos de los Cetáceos:] • Las extremidades anteriores están transformadas en aletas que funcionan a modo de timones (ilustr.
Comportamiento del traductor no especialista Cabe juzgar como traductor no especialista de un texto didáctico o divulgativo aquel que es lego, que carece de formación específica, en la disciplina y campo correspondientes a ese texto de partida. Aunque, en principio, para la traducción de un texto científico-técnico didáctico o divulgativo, un traductor no especialista se encuentra en clara desventaja en relación con el traductor especialista o semiespecialista (categorías de traductor cuyos contingentes son exiguos), aquí nos proponemos mostrar, a propósito de la deficiencia factual presente en el ej. [2a], que, en la práctica, un traductor no especialista con sólida cultura general y rigurosa formación profesional (en el campo de la traducción) también está capacitado, es suficiente, para garantizar óptimos resultados en la traducción de textos científicotécnicos didácticos y, sobre todo (por su menor especialización), divulgativos.

En esta línea, no se debe perder de vista, en primer lugar, que los textos científico-técnicos de carácter extradisciplinar (didácticos y divulgativos) surgen en virtud, por un lado, de un proceso de desintensificación de las tendencias a la precisión, formalidad y coherencia propias de los textos especializados intradisciplinares (de investigación) y, por otro lado, de la incorporación de los recursos propios de los lenguajes pedagógico, periodístico y ensayístico, lo cual, en conjunto, facilita la inteligibilidad textual de un público lego y, por tanto, también la del traductor no especialista (Garrido, 2016: 67-IOI). En segundo lugar, debe tenerse en cuenta que el surgimiento de internet, de su universo textual

$623 \mathrm{~b}$ [en esta ilustración, que muestra la cintura escapular y el esqueleto braquial de un delfín, se ven 5 dígitos en la mano o autopodio]). Los dígitos ya no se pueden reconocer externamente. [...] - Hiperfalangia (incremento del número de falanges). El segundo dedo es por lo general el más largo (ilustr. 623b)». 
y de sus motores de búsqueda ha representado una revolución en el trabajo del traductor (no especialista), quien, ahora, con herramienta tan potente y ubicua, puede, con gran facilidad, documentarse y determinar equivalencias terminológicas.

Además, internet proporciona al traductor (no especialista) de textos científico-técnicos un recurso conceptual y terminológico valiosísimo, aún no suficientemente ponderado, para hacer frente, más allá de la determinación de equivalencias terminológicas, al desafío de máxima dificultad constituido por la eventual presencia en el texto de partida de errores factuales: la enciclopedia multilingüe Wikipedia. Frecuentemente despreciada debido, sobre todo, a un tratamiento pobre y deficiente, en algunas versiones lingüísticas importantes, de materias propias de las ciencias sociales y de las humanidades, al traductor (no especialista) de textos científico-técnicos didácticos y divulgativos le interesa saber que, por lo menos, las versiones inglesa y alemana de Wikipedia son, hoy en día, muy completas y rigurosas en los campos de las ciencias naturales, constituyéndose, así, en recurso fundamental para la detección de errores factuales del texto de partida. ${ }^{\text {II }}$

Así, si la sólida cultura general de que debe disponer el traductor no especialista (la cual tiene que incluir elementos científico-técnicos) no se revelase suficiente para que este detecte de forma inmediata la falsedad de la afirmación

II Esta valoración positiva nos la dicta nuestra experiencia como consultores habituales de Wikipedia, impulsada por nuestra dedicación a la traducción de textos científicos y por la curiosidad intelectual, pero también disponemos, en ese sentido, de referencias externas: así, en 2016 , el premio concedido a periodistas y escritores científicos por la Sociedad de Químicos Alemanes (Preis der Gesellschaft Deutscher Chemiker für Journalisten und Schriftsteller) lo ganó la Redacción de Química de la Wikipedia en lengua alemana.

de que «los cetáceos poseen más de cinco dedos en sus extremidades (anteriores)» (ej. [2a]) - lo que, siendo realistas, es probable-, o para que le asalten serias dudas acerca de la correspondiente veracidad, su formación como traductor — que ha de tener en cuenta las posibilidades ofrecidas por internet - debe impulsarlo a intentar documentar, aunque sea de forma fugaz, buena parte de los enunciados relevantes del texto de partida, entre los que, en nuestro caso, se encontrará el relativo al número de dedos de los cetáceos. En ese sentido, el recurso a la enciclopedia internética Wikipedia resulta, por su gran eficacia, indispensable, y así lo vemos también en nuestro caso concreto.

En efecto, el artículo «Cetacea» de la versión inglesa y el artículo «Wale» de la versión alemana de Wikipedia (consulta: i2.5.2017) incluyen dos ilustraciones que claramente atestiguan la presencia de cinco dedos en los miembros anteriores de los cetáceos ${ }^{\mathrm{I2}}$ (las tituladas «Cetacea skeletons» [v. infra ilustración] y «Comparison of the skeleton of Dorudon atrox and Maiacetus inuus in swimming position», en el primer caso, $\mathrm{y}$ «Skelett eines Bartenwals (ohne Barten)» [v. infra ilustración] y «Vergleich des Skeletts von Dorudon

I2 De hecho, este elemento gráfico contribuye a afianzar el rigor de la información proporcionada aquí por Wikipedia. También en la divulgativa Enciclopedia National Geographic de los animales (Cooke et al., 2017) se observa, en sendas ilustraciones del esqueleto, la presencia de cinco dedos en las extremidades anteriores de los Odontocetos (pág. 178) y en las de los Misticetos (pág. 185). Además, en la pág. I78, se puede leer: «[En los odontocetos] Las extremidades posteriores desaparecieron, las anteriores se transformaron en aletas, si bien aún conservan los huesos de cinco dedos». En cualquier caso, la observación del resultado de introducir las palabras clave «cetáceos, esqueleto» en el Google Imágenes (importantísimo complemento de Wikipedia) permite comprobar que los cetáceos tienen 4 o 5 dedos en las extremidades anteriores. 
atrox und des Protocetiden Maiacetus inuus in Schwimmhaltung», en el segundo). ${ }^{13}$

Podemos decir, por tanto, que, pertrechado con este potente recurso, y concienciado de la necesidad de su utilización, el traductor no especialista de textos didácticos o divulgativos no se encuentra, en absoluto, inerme para detectar y corregir buena parte de las deficiencias factuales que eventualmente presenten sus textos de partida, lo cual, sumado a la posibilidad de una intervención complementaria de un revisor científico (no indispensable), en nuestra opinión, hace del traductor no especialista, mas sí verdaderamente competente, una buena opción, una opción aceptable, para verter textos científico-técnicos didácticos y divulgativos.

I3 En este caso, el artículo correspondiente en español («Cetacea») carece de esas ilustraciones esclarecedoras. En cuanto al componente verbal de estos artículos, la naturaleza no hiperdactílica de los cetáceos (o, de hecho, su pentadactilia o, secundariamente, su tetradactilia) solo figura en él de forma implícita (búsquedas automáticas de los términos ES dedo, EN finger y DE Finger): «Habiendo derivado de la de los mamíferos terrestres, la pata posterior [sic: por anterior] de los cetáceos se compone de los mismos huesos: el húmero, el radio y el cúbito. Sin embargo, estos huesos son más cortos y más planos que en los mamíferos terrestres y el cúbito y el radio son más largos que el húmero. Todos los cetáceos presentan un cierto grado de hiperfalangia, especialmente en los dedos centrales. El mayor número de falanges se da en los calderones negros, que tienen entre 3 y 4 en el primer dedo, entre 9 y I4 al segundo y entre 9 y II el tercero»; "The front limbs are paddle-shaped with shortened arms and elongated finger bones, to support movement. They are connected by cartilage. The second and third fingers display a proliferation of the finger members, a so-called hyperphalangy.»; «Die vorderen Gliedmaßen sind paddelförmig mit verkürzten Arm- und verlängerten Fingerknochen, um die Fortbewegung zu unterstützen. Sie sind durch Knorpel verwachsen. Am zweiten und dritten Finger kommt es zudem zu einer Vermehrung der Fingerglieder, einer so genannten Hyperphalangie».
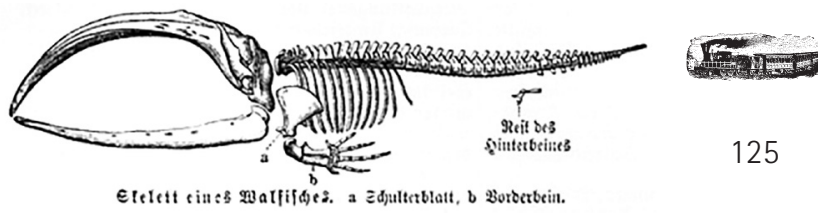

Ilustración que muestra el esqueleto de una ballena con barbas (misticeto), presente en los artículos «Cetacea», de Wikipedia-en, $y$ "Wale», de Wikipedia-de. Se aprecia la pentadactilia de la extremidad anterior (el "espolón" que parece surgir en la base del quinto dedo corresponde al hueso pisiforme, del carpo).

\section{EL TRADUCTOR ESPECIALISTA Y LO INACEPTABLE}

Caracterizadas las posibilidades de actuación del traductor especialista, del traductor semiespecialista y, sobre todo, del traductor no especialista frente al error factual del texto base científico-técnico de carácter extradisciplinar, ahora analizamos, por su gran interés para la traducción de textos didácticos y divulgativos, una nociva tendencia que hemos venido notando a lo largo de los años en las traducciones de libros de texto y de artículos y libros de divulgación del campo de la biología realizadas por traductores especialistas en alguna disciplina biológica. Paradójicamente, esa tendencia consiste en que demasiados de esos traductores especialistas traducen mal, en textos de biología, términos del campo de la biología. En concreto, se observa que, con relativa frecuencia, un traductor especialista en alguna de las disciplinas biológicas de carácter fundamental (bioquímica, citología, embriología, fisiología, inmunología, genética, etc.) vierte de forma errónea, o incluso indebidamente omite en el texto meta, términos propios de la biología sistemática (taxonomía) $y$, en especial, las denominaciones de grupos de organismos, componente terminológico, este, muy frecuente y relevante en los textos didácti- 
cos y divulgativos de los campos de la biología y de la medicina (Garrido, 2016: 204-209).

Las razones que explican esta nociva tendencia, como vamos a ver, pueden identificarse, de modo genérico, con un punto débil de la figura del traductor especialista y, de modo específico, con una inaceptable actitud de desdén intelectual. Así, según hemos declarado en el punto 2.2.I, los textos científico-técnicos didácticos y divulgativos, por su frecuente carácter integrador, suelen manejar términos y conceptos pertenecientes a diversas disciplinas de un determinado campo (o, incluso, a diferentes campos), por lo que el traductor especialista (en una disciplina concreta), en la práctica, debe lidiar con (algunos) problemas de determinación de equivalentes terminológicos similares a los que enfrenta un traductor semiespecialista o no especialista (por ejemplo, un traductor especialista en zoología, para traducir un término de fisiología vegetal en un libro divulgativo del campo de la ecología, tendrá que realizar un esfuerzo de documentación similar al de un traductor con conocimientos generalistas de biología o, incluso, al de uno carente de formación específica en biología).

$\mathrm{Si}$, por consiguiente, la condición de traductor especialista frecuentemente no exime de la necesidad de realizar esfuerzos de documentación al traducir textos didácticos y divulgativos, en el caso de los traductores especialistas en disciplinas de biología fundamental que vierten textos de biología de enfoque integrador, esa exigencia es, con excesiva frecuencia, negligentemente desconsiderada en relación, sobre todo, con términos y conceptos propios de la taxonomía, o sistemática, botánica y zoológica. Para entender este fenómeno, que puede perjudicar lastimosamente el valor de una traducción y que denota cierta falta de honradez intelectual, ha de saberse que, a partir del nacimiento de

la biología moderna, producido en la segunda mitad del siglo xix y en los primeros decenios del xx (con el progreso de la fisiología, la formulación y perfeccionamiento de la teoría evolutiva y el desarrollo de la genética, la bioquímica y la biología celular), y debido a un relativo estancamiento inicial (teórico y metodológico) de las disciplinas clásicas botánica, zoología y taxonomía (biología sistemática) —eficazmente superado a partir de la segunda mitad del siglo $\mathrm{xx}$-, está algo extendida entre los practicantes de las disciplinas de la biología fundamental una infundada actitud de superioridad intelectual hacia los practicantes de la biología sistemática y cierta injustificable y dañina minusvaloración de esas materias. ${ }^{\mathrm{I}}$

Con el ánimo de llamar la atención sobre este injustificable proceder de algunos traductores especialistas, y de estimular la adopción de buenas prácticas al respecto, a seguir aducimos una serie de ejemplos de traducciones de textos didácticos y divulgativos del campo de la biología que contienen flagrantes errores factuales en el tratamiento de términos zoológicos y botánicos. Los ejemplos [3a-c] y [4] corresponden a un libro de texto y a un artículo de divulgación vertidos al castellano por traductores especialistas en bioquímica y en ecología, respectivamente, y los ejemplos [5a-c] y [6a-c] corresponden a una monografía didáctica y a un libro de divulgación vertidos al gallego-portugués por traductores especialistas en genética y en embriología, respectivamente: ${ }^{15}$

I4 Esta es una impresión adquirida por el autor de estas líneas desde dentro, ya que este cuenta con formación no solo en el campo de la traducción, sino también en el de la biología, habiéndose doctorado en el área de la zoología (sistemática). En relación con el relativo estancamiento padecido a principios del siglo xx por la biología sistemática, y en relación con su posterior aggiornamento, puede consultarse, por ejemplo, Hennig (I999: I-Io).

${ }_{15}$ Obsérvese, por cierto, que esta nociva tendencia 
[3a] Lehninger - Principios de Bioquímica: I36-I37: «Figura 6-I4 [en esta ilustración las aves mencionadas son pingüino, pollo, pavo y pato]: (a) Localización de los 27 residuos invariables en el citocromo $c$ de unas 60 especies, entre las que se encuentran mamíferos, peces, reptiles, anfibios, pájaros [orig. EN birds], insectos y otros invertebrados, hongos y plantas».

COMENTARIO: Ninguna de las aves mencionadas en esta ilustración es un pájaro, o sea, ninguna pertenece al grupo (orden) Passeriformes. La confusión entre los términos pájaro y ave es impropia de un texto de biología, y un error frecuente de los traductores de textos divulgativos (Garrido, 20I6: 173-I76).

[3b] Lehninger-Principios de Bioquímica: $38 \mathrm{r}$ : «Figura I4-II [en esta ilustración se ve un guepardo, especie Acinonyx jubatus]: Los potentes y muy coordinados músculos esqueléticos del leopardo [especie Panthera pardus] le permiten alcanzar una velocidad de unos $130 \mathrm{~km} / \mathrm{h}$ durante unos centenares de metros».

[3c] Lehninger - Principios de bioquímica: 387 [cuadro de texto I4-3]: «Figura I [en esta ilustración se ve una luciérnaga, insecto coleóptero]: La libélula [insecto odonato], una especie de coleóptero».

[4] Scientific American, I2/1996: 60: "Jaap Graveland and his colleagues at the University of Groningen have noted that certain

también puede estar detrás de la deficiencia factual de Evolution: A Very Short Introduction que aquí analizamos, pues ese error se refiere a un concepto de zoología (sistemática), y los autores y los revisores de ese texto son, todos, especialistas en (sub)disciplinas de biología fundamental (B. y D. Charlesworth, en genética). De hecho, el dato falso sobre los dedos de los cetáceos tal vez no constituya un mero lapso léxico, ya que la segunda referencia (falsa) de ese fagmento a la presencia de más de cinco dígitos en los cetáceos - que se vincula a la configuración de las extremidades en los vertebrados terrestres más antigos (y en los peces) - parece apuntar a que se trata de un error más profundo, de naturaleza conceptual. birds, such as the great tits of the Netherlands, produce thinner, more fragile eggs in forests that have been heavily damaged by acid rain and have low stores of calcium in the soil.»

Investigación y Ciencia, 2/1997: 58: «El grupo dirigido por Jaap Graveland, de la Universidad de Groningen, ha observado que los grandes herrerillos de Holanda producen huevos más frágiles en los bosques afectados por la lluvia ácida y con bajas reservas de calcio en el suelo».

comentario: Aquí se ha utilizado como equivalente castellano de great tit (que corresponde a la especie Parus major) el indebido calco * gran herrerillo, que no tiene sentido zoológico, pues la denominación vernácula real de esa especie en castellano es carbonero común (Peterson et al., I989: 236).

[5a] The Growth of Biological Thought. Diversity, Evolution, and Inheritance: 246 : «A specialist of the taxonomy of mites (Acarina), nematodes, spiders [...].»

Historia do Pensamento Biolóxico. Diversidade, Evolución, Herdanza: 301: «Un especialista na taxonomía dos ácaros, dos nematodos, dos arácnidos $[\ldots]$...

COMENTARIO: Los ácaros mencionados en este pasaje son arácnidos del grupo (orden) Acarina, mientras que el término inglés spiders (literalmente, 'arañas') corresponde a arácnidos del grupo (orden) Araneae, por lo que la equivalencia correcta hubiese sido, no arácnidos, sino araneidos.

[5b] The Growth of Biological Thought. Diversity, Evolution, and Inheritance: 432: «[...] the agnath fishes (lampreys and hagfishes) [...].»

Historia do Pensamento Biolóxico. Diversidade, Evolución, Herdanza: 507: "[...] os peixes [= ES peces] agnatos (lampreas e similares) [...]». 
COMENTARIO: El término inglés hagfish representa la denominación vernácula de un grupo concreto de craneados primitivos (agnatos) conocidos en castellano como mixinos o peces bruja (en gallego[-portugués] como peixesbruxa, enguias-de-casulo, feiticeiras o mixinas). En este caso, el traductor optó por una indebida omisión de esa referencia taxonómica («lampreas y similares»), que empobrece el contenido informativo del texto de llegada respecto al original.

[5c] The Growth of Biological Thought. Diversity, Evolution, and Inheritance: 45r: «Groups with a relatively low active dispersal ability, like most terrestrial mammals, true freshwater fishes, or earthworms, have very different patterns from those of easy dispersers like freshwater plankton, ballooning spiders, birds, and some groups of insects.»

Historia do Pensamento Biolóxico. Diversidade, Evolución, Herdanza: 527: «Os grupos dotados dunha capacidade de dispersión relativamente cativa [= ES pequeña], como o caso dos máis dos mamíferos terrestres, os peixes de auga doce $[=\mathrm{ES}$ peces de agua dulce], ou os vermes terrestres [= ES gusanos terrestres], teñen distribucións ben distintass $[s i c]$ das dos grupos que se dispersan de modo máis doado [= Es fácil], o plancto de auga doce, algunbas arañas, os paxaros e algúns grupos de insectos».

comentario: Este fragmento está repleto de dislates en la traducción de los términos zoológicos. En primer lugar, la expresión original true freshwater fishes, incorrectamente vertida como peixes de auga doce (= Es peces de agua dulce), se refiere, en realidad, a peces exclusivamente dulceacuícolas (obsérvese que "peces de agua dulce», sin más especificación, son, por ejemplo, el salmón y la anguila, que, por ser también marinos, presentan una elevada capacidad de dispersión); en segundo lugar, la equivalencia

correcta de earthworms es, en gallego-portugués, minhocas (= Es lombrices de tierra) u oligoquetas (= Es oligoquetos), no vermes terrestres (= Es gusanos terretres), pues "gusanos terrestres» son, por ejemplo, ciertos nematodos cosmopolitas que presentan una gran capacidad para la dispersión pasiva por mediación de aves; en tercer lugar, ballooning spiders no corresponde a «algunas arañas», sino, concretamente, a arañas que, suspendidas en la atmósfera, integran el llamado plancton aéreo, es decir, arañas que presentan capacidad de aerostación; por último, los pájaros, en los textos de biología, son aves del orden Passeriformes, pero en este pasaje birds (que equivale a aves) también incluye, por ejemplo, a las Caradriiformes, que no son pájaros.

[6a] Oaxaca Journal: 8-9: «We also had a glassed-in conservatory, always warm and humid, where a great tassel fern hung, and delicate filmy ferns and tropical ferns could be grown.»

Diário de Oaxaca: 23: «Também tínhamos uma estufa [= ES invernadero] toda coberta de vidro, sempre quente e húmida, onde estava pendurado um Polystichum polyblepharum ${ }^{4}$ grande, e onde podíamos cultivar fetos [= ES helechos] delicados e tropicais. [...] [nota de la traductora:] 4 Sem nome em português; designado em inglês por "tassel fern"».

COMEntario: En inglés, filmy fern no equivale a «helecho», como pretende aquí la traductora, sino a «helecho de la familia Himenofiláceas», por lo que una traducción correcta de «delicate filmy ferns» hubiese sido «delicados fetos [= ES belechos] himenofiláceos».

[6b] Oaxaca Journal: 32: «Like potatoes, they [los tomates] are in the Solanaceae family, a family full of particularly deadly plants, including the thorn apple and henbane.» 
Diário de Oaxaca: 42: «Tal como as batatas, [los tomates] pertencem à família dos Solenáceos, que está cheia de plantas particularmente mortíferas, incluindo a maçã espinhosa [literalmente, ES «manzana espinosa»] e o meimendro negro [= ES beleño negro]».

COMENTARio: En primer lugar, en portugués, como en castellano, el término correcto (designativo de familia de plantas) no es *Solanáceos, sino Solanáceas; en segundo lugar, el término inglés thorn apple no equivale, ni en portugués ni en castellano, al calco espurio «manzana espinosa», sino a estramonio (= PT estramónio).

[6c] Oaxaca Journal: 48: "Other yellow flowers are dismissed as DYCs-damned yellow composites.»

Diário de Oaxaca: 54: «Outras flores amarelas som despachadas para o saco das DYC Damned Yellow Composites ${ }^{16}$. [...] [Nota a pie de página:] ${ }^{16}$ Qualquer coisa como "malditos compósitos amarelos" (N. T.)».

comentario: En el pasaje original, composites designa, claramente, un grupo de plantas, por lo que la equivalencia correcta no es compósitos, sino PT compostas (= ES compuestas).

\section{CONCLUSIONES}

Como hemos visto a lo largo del presente artículo, en los textos (científico-técnicos) didácticos y divulgativos contemporáneos con cierta frecuencia surgen deficiencias factuales, por mucho que aquellos hayan sido producidos por autores reputados, revisados por especialistas y publicados en medios y editoriales de prestigio. Cuando tales documentos aquejados de alguna deficiencia factual sirven como texto de partida de una traducción, es responsabilidad del traductor, en el marco de la traducción comu- nicativa, detectar esos errores $y$, mediante la incorporación de la correspondiente corrección, no trasladarlos a su texto de llegada, lo cual representa uno de los mayores desafíos para los traductores de este tipo de textos.

Entre los traductores capacitados para la realización de (buenas) traducciones de textos científico-técnicos de carácter didáctico o divulgativo, pueden distinguirse tres categorías en función del respectivo grado de especialización en la disciplina a que se adscribe el texto de partida: el traductor especialista, el traductor semiespecialista y el traductor no especialista. Como en el presente artículo se muestra a propósito de un error factual concreto presente en un libro divulgativo inglés del campo de la biología, aunque, al verter textos científico-técnicos didácticos y divulgativos, el traductor especialista y el traductor semiespecialista, por su mayor bagaje de conocimientos de especialidad, tienen, en general, más facilidad que el traductor no especialista para detectar errores factuales en el texto base y corregirlos en el texto meta (como, igualmente, para determinar equivalencias terminológicas), hoy en día este último traductor, sin embargo, no se encuentra inerme, en absoluto, para hacer frente a ese importante desafío. En efecto, con el advenimiento de internet, el traductor no especialista de textos científicotécnicos didácticos y divulgativos ha pasado a contar, entre otros potentes recursos, con una fuente de consulta tan accesible y tan rica y rigurosa en el ámbito de las ciencias naturales como, por lo menos, las versiones en lenguas inglesa y alemana de la enciclopedia Wikipedia (valoración positiva, esta, confirmada, de hecho, en el caso concreto de traducción aquí estudiado). Por consiguiente, si, en principio, la figura del traductor especialista resulta ideal para la realización de traducciones de textos científicotécnicos de carácter didáctico o divulgativo, y 
muy favorable la del traductor semiespecialista, el traductor no especialista bien formado y convenientemente concienciado de que puede y debe compulsar (en la Wikipedia inglesa o alemana, con el respaldo ulterior de otras fuentes) buena parte de los enunciados relevantes de su texto de partida, se podría revelar suficiente, como aquí se ve, para abordar con garantías una empresa semejante.

Por otro lado, aunque el traductor especialista (en una determinada disciplina o subdisciplina) se revela, en principio, idealmente capacitado para verter textos (científico-técnicos) didácticos y divulgativos, nuestro trabajo señala que ese tipo de traductor no deja de presentar algún punto débil en el marco de esos encargos. Concretamente, se apunta la circunstancia, de carácter genérico, de que uno de tales traductores nunca podrá ser verdaderamente experto en las varias disciplinas, o incluso campos, que, por su frecuente enfoque integrador, suelen abordar los textos científico-técnicos didácticos y divulgativos; además, como aquí se pone de manifiesto, en el caso específico de los traductores especialistas en alguna disciplina o subdisciplina de la biología fundamental que traducen textos didácticos o divulgativos del campo de la biología se aprecia que, con bastante frecuencia, tratan de forma negligente, $y$ vierten mal, términos propios de la biología sistemática (taxonomía botánica y zoológica), nociva tendencia, esta - probablemente derivada de una injustificable actitud de desdén intelectual-, que el presente artículo documenta mediante ejemplos elocuentes.

En cualquier caso, en una época como la actual, en que los ubicuos traductores automáticos están haciendo notables progresos y alcanzando resultados cada vez mejores, tanto si se trata de un traductor especialista como de un traductor no especialista, es indispensable que ese traductor

bumano de textos didácticos y divulgativos, para no revelarse superfluo o redundante, sea verdaderamente competente, $\mathrm{y}$, por tanto, que sea capaz, entre otras cosas, de hacer frente al desafío planteado por la eventual presencia de errores factuales en el texto de partida. Estúdiese, si no, a modo de referencia final, la versión que de nuestro fragmento textual [2a] nos ofrece hoy en día uno de esos traductores automáticos:

[2c] Traducción efectuada por Google Translate (junio de 2or7; subrayados nuestros): «Otro conjunto de hechos que apoya fuertemente la teoría de la evolución es proporcionado por modificaciones de la misma estructura en diferentes especies. Por ejemplo, los huesos de las alas de los murciélagos y de los pájaros indican claramente que son miembros modificados, aunque parezcan muy diferentes de los antebrazos de otros vertebrados (Figura гв). Del mismo modo, aunque las aletas de las ballenas se parecen mucho a las aletas de los peces, y están claramente también bien adaptadas para la natación, su estructura interna es como los pies de otros mamíferos, a excepción de un número creciente de digitos. Esto tiene sentido, dadas todas las otras pruebas de que las ballenas son mamíferos modificados (por ejemplo, respiran con pulmones y amamantan a sus crías). La evidencia fósil muestra que los dos pares de extremidades de los vertebrados terrestres se derivan de los dos pares de aletas de los peces lóbulos finos (de los cuales los celacantos son los representantes vivos más famosos, véase el capítulo 4). De hecho, los primeros fósiles de vertebrados terrestres tenían más de cinco dígitos en sus miembros, al igual que los peces y las ballenas».

RECIBIDO EN JUNIO DE 2017 ACEPTADO EN OCTUBRE DE 2018 VERSIÓN FINAL DE OCTUBRE DE 2018 


\section{BIBLIOGRAFÍA}

\section{Referencias citadas}

Beaumont, André y Pierre Cassier (1987). Biologie animale. Les Cordés, Anatomie comparée des Vertébrés, 6. ${ }^{a}$ ed., París: Dunod.

Cooke, Fred, Hugh Dingle, Stephen Hutchinson, George McKay, Richard Schodde, Noel Tait y Richard Vogt (dir.) (2017). Enciclopedia National Geographic de los animales, 2. ${ }^{\mathrm{a}}$ ed., trad. anónima de The Encyclopedia of Animals: A Complete Visual Guide, Barcelona: RBA Libros.

Cooper, Lisa Noelle; Berta, Annalisa; Dawson, Susan D.; Reidenberg, Joy S. (2007). «Evolution of Hyperphalangy and Digit Reduction in the Cetacean Manus», The Anatomical Record, 290, $654-672$.

Fleischmann, Eberhard y Peter A. Schmitt (2004). «Fachsprachen und Übersetzung», en Harald Kittel, Armin Paul Frank, Norbert Greiner et al. (dir.), Übersetzung / Translation / Traduction. Handbücher zur Sprach- und Kommunikationswissenschaft, n. ${ }^{\circ}$ 26.r. Berlín/Nueva York: Walter de Gruyter, 531-542.

Garrido, Carlos (2015a). «Deficiencias del texto de partida en la traducción de textos destinados a la enseñanza y divulgación de la ciencia», Meta, 6o/3, 454-475.

- (2015b). «Tres persistentes falacias zoológicas, reverberadas/debeladas en la traducción de textos científicos de carácter didáctico»,Panace@. Revista de Medicina, Lenguaje y Traducción, 4I, 60-72.

- (20I6). A Traduçom do Ensino e Divulgaçom da Ciência, Vigo: Servizo de Publicacións da Universidade de Vigo.

Hennig, Willi (I999 [ig66]). Phylogenetic Systematics, reimpr. $3 \cdot{ }^{\mathrm{a}}$ ed., trad. D. Dwight Davis y Rainer Zangerl, Urbana: University of Illinois Press.

Horn-Helf, Brigitte (I999). Technisches Übersetzen in Theorie und Praxis, Tubinga: A. Francke Verlag.

Kyrieleis, Armin y Hannes Paulus (2000). «Extremitäten», en Rolf Sauermost (dir.), Lexikon der Biologie, Heidelberg: Spektrum Akademischer Verlag/Elsevier, s.v.

Liem, Karel F.; Bemis, William E.; Walker, Warren F.; Grande, Lance (3200I). Functional Anatomy of the Vertebrates. An Evolutionary Perspective, Belmont: Thomson/Brooks/Cole.
Mickoleit, Gerhard (2004). Phylogenetische Systematik der Wirbeltiere, Múnich: Verlag Dr. Friedrich Pfeil.

Peterson, Roger; Mountfort, Guy; Hollom, P. A. D. (I989). Guía de campo de las aves de España y de Europa, trad. Mauricio González Díez, Barcelona: Ediciones Omega.

SANDER, Martin (2004). "†Ichthyosauria, Fischechsen», en Wilfried Westheide y Reinhard Rieger (ed.), Spezielle Zoologie. Teil 2: Wirbel-oder Schädeltiere, Heidelberg: Spektrum Akademischer Verlag, 352-353.

Schmitt, Peter A. (I999a). Translation und Technik, Tubinga: Stauffenburg Verlag.

- (I999b). «Defekte im Ausgangstext», en Mary Snell-Hornby, Hans G. Hönig, Paul Kußmaul y Peter A. Schmitt (ed.), Handbuch Translation, 2. ${ }^{\text {a }}$ ed., Tubinga: Stauffenburg Verlag, I47-I5I.

Schultze, Hans-Peter y Rainer SсHоch (2004). «Tetrapoda, Landwirbeltiere», en Wilfried Westheide y Reinhard Rieger (ed.), Spezielle Zoologie. Teil 2: Wirbel-oder Schädeltiere, Heidelberg: Spektrum Akademischer Verlag, 303-306.

\section{Fuentes de los ejemplos}

Charlesworth, Brian y Deborah Charlesworth (2003). Evolution: A Very Short Introduction, serie Very Short Introductions, n. ${ }^{\circ}$ Ioo, Oxford: Oxford University Press.

Hedin, Lars O. y Gene E. Likens (i996). «Atmospheric Dust and Acid Rain». Scientific American, I2/1996, 56-6o.

- (I997). «Polvo atmosférico y lluvia ácida», trad. Manuel Puigcerver, Investigación y Ciencia, 2/1997, 54-59.

Lehninger, Albert L. (1984). Principios de Bioquimica, trad. Jorge Bozal Fes y Antonio Cortés Tejedor. Barcelona: Ediciones Omega.

Liberman, Charles M. (2015a). «Hidden Hearing Loss», Scientific American, 8/2015, 39-43.

- (2015b). «Sordera oculta», trad. Andrés Martínez, Investigación y Ciencia, Io/2015, 64-69.

Mayr, Ernst (I982). The Growth of Biological Thought. Diversity, Evolution, and Inheritance, Cambridge (Massachusetts): The Belknap Press of Harvard University Press.

- (1998). Historia do Pensamento Biolóxico. Diversidade, Evolución, Herdanza, trad. Emilio Valadé 
del Río, colección «Lingua Franca», n. ${ }^{\circ}$ 4, Santiago de Compostela: Servizo de Publicacións da Universidade de Santiago de Compostela.

SAcks, Oliver (2002). Oaxaca Journal, Nueva York: Vintage Books.
- (2orr). Diário de Oaxaca, trad. Clara Pinto Correia, Lisboa: Relógio D’Água. 